\title{
Bactericidal capacity of erythrocytes in human cardiovascular system
}

\begin{abstract}
Leukocytes cannot recognize or engulf bacteria in flowing blood and so phagocytosis is impossible in the bloodstream. Catching by electric charge and killing by oxidation is the most rapid and rational bactericidal mechanism in the bloodstream. Erythrocytes are well equipped for using this mechanism: their unique membrane is easily charged by friction and so effectively attracts and fixes bacteria; their inner space contains enough oxygen for oxidation and prompt killing of bacteria. Erythrocytes kill bacteria but cannot digest them. The dead bacteria are disintegrated and digested in the liver and the spleen.
\end{abstract}

Volume 2 Issue 5 - 2016

\author{
Hayk Minasyan \\ Private Laboratory, Armenia
}

Correspondence: Hayk Minasyan, Mamikonyanz 38-38, Yerevan, Armenia, 00 I4, Tel +374-772-552-95,

Email haykminasyan@rambler.ru

Received: June 20, 2016 | Published: July 22, 2016

Keywords: bacteremia, erythrocyte, bloodstream

\section{Mini review}

Fluid dynamics influences innate cellular immunity. In the human body fluid dynamics is different: the blood rapidly moves in the cardiovascular system whereas lymph, interstitial and cerebrospinal fluids move slowly. Bactericidal mechanisms of the body depend upon the speed of liquid media flow. Phagocytosis of bacteria needs relatively long time ${ }^{1,2}$ and so it occurs in slow motion liquid media, for example, in the tissues and lymph. In the bloodstream the cells move rapidly and only electric charge attraction phenomenon can pull and fix bacteria on the surface of bactericidal cells. In the tissues resident macrophages, lymph node leukocytes and neutrophils transmigrated from the bloodstream are the main bactericidal cells; in the bloodstream the main bactericidal cells are erythrocytes. ${ }^{3,4}$ They catch bacteria by electric charge and kill by oxygen release. ${ }^{5}$ The net electrical charge of molecules exposed at the surface of erythrocyte cell membrane (zeta potential) is- 15.7 millivolts $(\mathrm{mV})^{6}$ and is not enough for attraction and fixation of bacteria. Enough strong charge for catching and fixing bacteria is generated during erythrocytes move in the cardiovascular system. This charge is called triboelectric because it is induced by friction: rubbing of erythrocytes against each other and vessel walls. The blood flow provides additional triboelectric charging that depends upon blood viscosity, hematocrit, and the speed and manner of blood flow (laminar, turbulent, etc.). ${ }^{5}$ Bacteria are triboelectrically charged as well and this charge considerably exceeds bacterial own zeta potential. In capillaries, the blood flow is relatively slow. Erythrocyte width is $25 \%$ larger than capillary diameter, ${ }^{7}$ and this causes elastic deformation of erythrocytes and friction against the walls of capillaries. The stimulation of membrane receptors by friction, triboelectric charge and membrane mechanical deformation cause abundant release of oxygen from oxyhemoglobin that improves the oxygen transfer from erythrocytes to tissues. The release of oxygen kills bacteria that pass the capillaries with erythrocytes, besides, bacteria are killed by mechanical squeezing between erythrocytes. Another co-factor for the oxygen release is osmotic pressure. The decreased velocity of flow in the capillaries increases the blood pressure, due to Bernoulli's principle. This induces gas and nutrients to move from the blood to the cells, due to the lower osmotic pressure outside of the capillary. The opposite process occurs when the blood leaves the capillaries and enters the venules, where the blood pressure drops due to an increase in flow rate. ${ }^{8}$
The cardiovascular system of the human body can be compared with a network of tubes. It consists of a pump and a system of branched vessels. The arteries transport the blood to the periphery in a manner similar to that of a water supply network. ${ }^{9}$ Rubbing of erythrocytes against each other and vessel walls generates triboelectric charging and is determined by the diameter of vessels and blood flow type and velocity. The diameter of blood vessels ranges from 8 micrometer to 34,000micrometer (Figure 1). Capillaries have the smallest diameter (8-10 micrometers), the largest diameter is in the V. Cava. The diameter of blood vessels is affected by different factors including vessel contraction and relaxation, development of atherosclerosis, presence of aneurisms and even respiratory cycle (as in V. cava inferior). Generally, blood flow is laminar (Figure 2). But in high speed flow, for example, in the ascending aorta, the flow may become turbulent. In this case, blood does not flow linearly and smoothly in adjacent layers, and becomes chaotic. Turbulent flow also happens at branch points of large arteries, in arterial stenosis, and across stenotic heart valves. Turbulence increases the energy needed to drive blood flow because of friction, which generates triboelectric charging of erythrocytes. Turbulence starts in high velocity of flow. So, increase in turbulence is not gradual. The features of the microscale flow for turbulent flows have not been revealed. In capillary tubes, the erythrocytes are more concentrated towards the centre of the vessel, leaving significant RBC-free layer near the vessel walls. ${ }^{10}$ This phenomenon is called the Fahraeus effect and is described as the decrease in average concentration of red blood cells in human blood as the diameter of the glass tube in which it is flowing decreases. The Fåhræus effect take place in blood vessels with diameters less than 500 micrometers. The Fahraeus effect occurs because the average $\mathrm{RBC}$ velocity is higher than the average plasma velocity.

Different diameter of the vessels in human circulatory system and high velocity of bloodstream provide friction of erythrocytes and their triboelectric charging in all parts of cardiovascular system. It means that erythrocytes are able to attract and fix bacteria during cycle of circulation. As a result, in arteries and capillaries erythrocytes catch bacteria and effectively kill them by oxidation (Figure 3 ). The situation is different with bacteria killing. After passing through capillaries and dropping oxygen to the tissues, erythrocytes lose capacity to kill bacteria. But the lack of oxygen short-term and lasts only during erythrocytes travel from venules to lung alveoli. Each 
complete circulation of erythrocytes takes about 20 seconds $^{11}$ and so erythrocytes are unable to kill bacteria for less than 20seconds. After entering the lungs and binding oxygen erythrocytes restore the capacity to kill bacteria. Pathogen catching by electric charge attraction is effective along human all blood vessels. A bacterium killing by oxidation is a universal and powerful bactericidal tool of innate immunity. Bacteria protect themselves from oxidation by producing catalase, thick and dense capsule and slowing down metabolic activity. The content of erythrocytes is bactericidal as well. Hemoglobin per se has broad-spectrum antimicrobial activity, ${ }^{12}$ it is a precursor of antibacterial peptides, ${ }^{13-15}$ and potentiates the activity of human defensins, cathelicidin and lysozyme. ${ }^{16}$

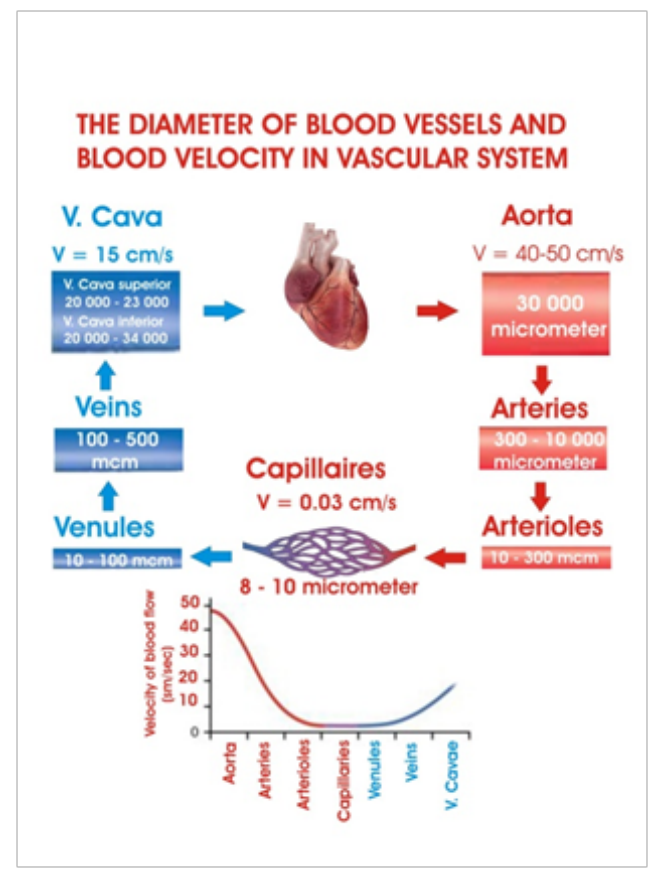

Figure 1 The diameter of blood vessels and blood velocity in vascular system.

\section{BLOOD FLOW IN THE DIFFERENT PARTS OF HUMAN VASCULAR SYSTEM}

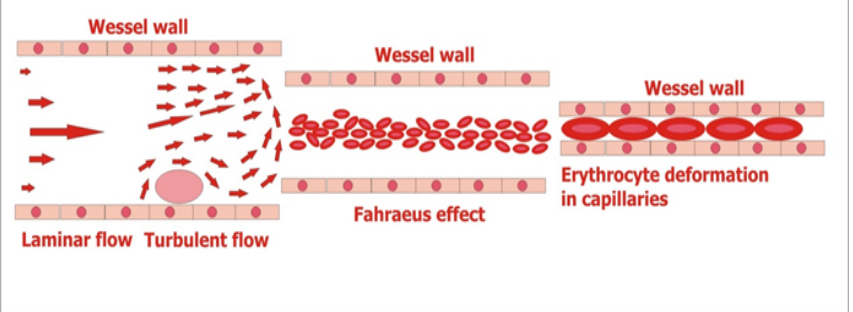

Figure 2 Blood flow in the different parts of human vascular system.

Another function of erythrocytes is the transport of immune complexes. ${ }^{17}$ Erythrocytes express complement receptors (E-CR), which can extrinsically bind $\mathrm{C} 3 \mathrm{~b}$ and $\mathrm{Cb} 4$. This interaction allows primate erythrocytes to bind complement opsonized particles and immune complexes, a phenomenon historically referred to as immune adherence. The binding of $\mathrm{C} 3 \mathrm{~b}$ and $\mathrm{C} 4 \mathrm{~b}$ by E-CR also leads to inhibition of complement activation. ${ }^{18}$ When antigen/antibody immune complex (IC) form in the presence of complement, C3b binds covalently to the complexes. Such opsonized complexes attach to cells bearing complement receptor $1(\mathrm{CRl})$, which is mostly found on erythrocytes in the circulation. This allows IC to be transported through the circulation to the fixed macrophage system of the liver and spleen. In addition, C3b bound to the complexes is catabolized by factor I (with $\mathrm{CRl}$ as a cofactor) so that the complement-activating properties of the complexes are reduced. Complement and erythrocyte CRl contribute to the safe and effective elimination of IC in humans. This physiologic system prevents IC deposition in the vessel walls. ${ }^{17}$ The loss of erythrocyte CR1 may be an important pathogenic factor in the development and severity of IC-mediated diseases, such as systemic lupus erythematosus (SLE). ${ }^{18}$

\section{ERYTHROCYTE BACTERICIDAL FUNCTION IN THE BLOODSTREAN}

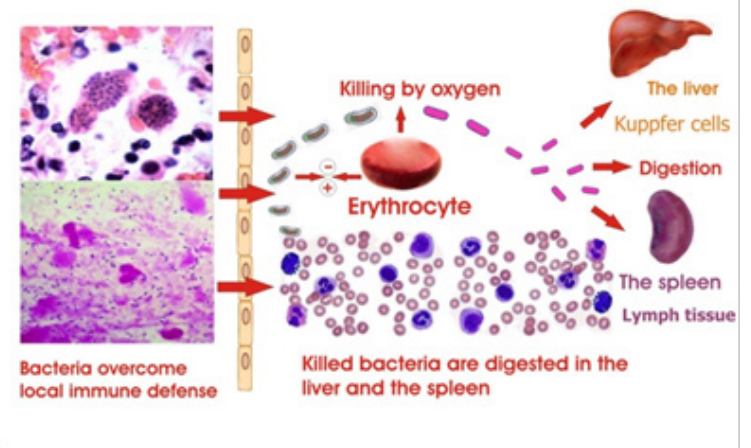

Figure 3: Erythrocyte bactericidal function in the bloodstream.

Erythrocyte number, intracellular amount of hemoglobin, abnormalities of erythrocyte structure and other changes in erythrocyte influence blood bactericidal activity. All kinds of anemia are associated with an increased frequency of bacterial infections. ${ }^{19,20}$ There is evidence to suggest that in hemolytic states massive erythrocyte ingestion by macrophages interferes with their antibacterial function, thereby predisposing infection. ${ }^{21,22}$ It often happens in the case of hemorrhage or erythrocyte extravagation, for example, in the stage of streptococcal pneumonia red hepatisation. ${ }^{23}$ In the tissues erythrocytes are void of oxygen and so are vulnerable to pathogens: bacteria destroy erythrocytes by hemolysins and/or enter and proliferate inside erythrocytes. ${ }^{23}$ Thus, erythrocytes may become a rich source of protein, iron and other nutrients for bacterial growth. The ingestion of erythrocyte by resident macrophages and transmigrated from the bloodstream leukocytes (neutrophils and monocytes) may inhibit or considerably decrease the Phagocytosis of pathogens.

In the bloodstream erythrocytes have enough oxygen for effective killing of bacteria, but they cannot destroy and digest them because of the absence of appropriate intracellular structures. Killed bacteria are digested in the liver and the spleen by Kuppfer cells and spleen macrophages.

\section{Conclusion}

The bactericidal activity of erythrocytes in the human cardiovascular system is provided by a unique physicochemical mechanism: erythrocytes attract (catch) bacteria by electric charge and kill by oxidation. Effective oxygen transport to distant tissues requires high speed of blood before capillaries and elastic deformation 
of erythrocytes in the capillaries with friction against their wall for maximal release of oxygen. As a result, erythrocytes become strongly charged and attract and fix by the charge bacteria on their surface. Effective oxygen transport also needs binding and delivery of maximal amount of oxygen by every red blood cell. So erythrocytes do not contain organelles and are full of hemoglobin that binds enough oxygen for intensive oxidation of bacteria. The bactericidal function of erythrocytes is determined by their main physiological function: oxygen supply and $\mathrm{CO}_{2}$ removal. So bacteria killing may be interpreted as a "side-effect" of erythrocyte oxygen transportation.

\section{Acknowledgements}

None.

\section{Conflict of interest}

The author declares no conflict of interest.

\section{References}

1. Lee CY, Herant M, Heinrich V. Target-specific mechanics of phagocytosis: protrusive neutrophil response to zymosan differs from the uptake of antibody-tagged pathogens. J Cell Sci. 2011;124(pt 7):1106-1104.

2. Herant M, Heinrich V, Dembo M. Mechanics of neutrophil phagocytosis: experiments and quantitative models. J Cell Sci. 2006;119(pt 9):19031913.

3. Minasyan H. Erythrocyte and blood antibacterial defense. Eur J Microbiol Immunol. 2014;4(2):138-143.

4. Minasyan H. Erythrocyte and leukocyte: two partners in bacteria killing. Int Rev Immunol. 2014;33(6):490-497.

5. Minasyan H. Mechanisms and pathways for the clearance of bacteria from blood circulation in health and disease. Pathophysiology. 2016;23(2):6166.

6. Tokumasu F, Ostera GR, Amaratunga C, et al. Modifications in erythrocyte membrane zeta potential by Plasmodium falciparum infection. See comment in PubMed Commons belowExp Parasitol. 2012;131(2):245-251.

7. Snyder, Gregory K, Sheafor, et al. Red blood cells: centerpiece in the evolution of the vertebrate circulatory system. Integrative and Comparative Biology. 1999;39(2):189-198.

8. Anthea M, Hopkins J, McLaughlin CW, et al. Human Biology and Health. Englewood Cliffs, USA: New Jersey: Prentice Hall; 1993.
9. Liepsch DW. Flow in tubes and arteries--a comparison. Biorheology. 1986;23(4):395-433.

10. Quinlan NJ. Mechanical loading of blood cells in turbulent flow. In: Barry Doyle, et al. editors. Computational Biomechanics for Medicine. galway, Ireland: Springer; 2014. p.1-13.

11. Pierigè F, Serafini S, Rossi L, et al. Cell-based drug delivery. Adv Drug Deliv Rev. 2008;60(2):286-295.

12. Liepke C, Baxmann S, Heine C, et al. Human hemoglobin-derived peptides exhibit antimicrobial activity: a class of host defense peptides. $J$ Chromatogr B Analyt Technol Biomed Life Sci. 2003;791(1-2):345-356.

13. Parish CA, Jiang H, Tokiwa $Y$, et al. Broad-spectrum antimicrobial activity of hemoglobin. Bioorg Med Chem. 2001;9(2):377-382.

14. Mak P, Wojcik K, Silberring J, et al. Antimicrobial peptides derived from heme-containing proteins: hemocidins. Antonie Van Leeuwenhoek. 2000;77(3):197-207.

15. Mak P, Wojcik K, Wicherek L, et al. Antibacterial hemoglobin peptides in human menstrual blood. Peptides. 2004;25(11):1839-1847.

16. Mak P, Siwek M, Pohl J, et al. Menstrual hemocidin HbB115-146 is an acidophilic antibacterial peptide potentiating the activity of human defensins, cathelicidin and lysozyme. Am J Reprod Immunol. 2007;57(1):81-91.

17. Pascual M, Schifferli JA. Another function of erythrocytes: transport of circulating immune complexes. Infusionsther Transfusionsmed. $1995 ; 22: 310-315$.

18. Birmingham DJ. Erythrocyte complements receptors. Crit Rev Immunol. 1995;15(2):133-154.

19. Berkowitz FE. Hemolysis and infection: categories and mechanisms of their interrelationship. Rev Infect Dis. 1991;13(6):1151-1162.

20. Kaye D, Hook EW. The influence of hemolysis on susceptibility to salmonella infection: additional observations. J Immunol. 1963;91:45184527.

21. Hand WL, King-Thompson NL. Effect of erythrocyte ingestion on macrophage antibacterial function. Infect Immun. 1983;40(3):917-923.

22. Hand WL. Inhibition of cell-free oxidative bactericidal activity by erythrocytes and hemoglobin. Infect Immun. 1984;44(2):465-468.

23. Minasyan H. Bacteria killer and bacteria pray. Int J Immunol. 2015;3(1$1): 1-7$. 